\title{
DEFORMATIONS OF HOMOMORPHISMS OF LIE GROUPS AND LIE ALGEBRAS
}

\author{
BY ALBERT NIJENHUIS ${ }^{1}$ AND R. W. RICHARDSON, JR. ${ }^{2}$
}

Communicated by E. Hewitt, September 8, 1966

The purpose of this note is to announce several results on deformations of homomorphisms of Lie groups and Lie algebras. Our main theorems are precise analogues of two basic theorems on deformations of complex analytic structures on compact manifolds, the rigidity theorem of Frölicher-Nijenhuis [3] and the local completeness theorem of Kuranishi [8]. In our tesults, sheaf cohomology is replaced by the cohomology of Lie groups and Lie algebras. Our proofs rely heavily on the theory of deformations in graded Lie algebras (GLA's) developed in [9]. Our results on Lie algebra homomorphisms follow immediately from the results given there, once the appropriate GLA is defined. Detailed proofs of the results on Lie group homomorphisms (which are only outlined here) will appear elsewhere.

1. Deformations of homomorphisms of Lie algebras. Let $\mathfrak{g}$ and $\mathfrak{h}$ be finite-dimensional real Lie algebras. For each integer $n \geqq 0$, let $E^{n}$ be the vector space of all alternating $n$-linear maps of $\mathfrak{g}$ into $\mathfrak{h}$; let $E=\oplus_{n} E^{n}$. We define a product on $E$, also denoted [, ] as follows: If $\phi \in E^{m}$ and $\psi \in E^{n}$, then $[\phi, \psi] \in E^{m+n}$ is given by

$$
\begin{aligned}
{[\phi, \psi]\left(x_{1}, \cdots, x_{m+n}\right) } & \\
& =\sum_{\sigma} \operatorname{sgn}(\sigma)\left[\phi\left(x_{\sigma(1)}, \cdots, x_{\sigma(m)}\right), \psi\left(x_{\sigma(m+1)}, \cdots, x_{\sigma(m+n)}\right)\right],
\end{aligned}
$$

where the sum is taken over all permutations $\sigma$ of $\{1, \cdots, m+n\}$ such that $\sigma(1)<\cdots<\sigma(m)$ and $\sigma(m+1)<\cdots<\sigma(m+n)$. When $\phi=\omega \otimes u$ and $\psi=\pi \otimes v$, where $\omega$ and $\pi$ are alternating (real-valued) forms on $g$ and $u, v \in \mathfrak{h}$, then $[\phi, \psi]=(\omega \wedge \pi) \otimes[u, v]$. Thus it follows easily that the product $(\phi, \psi) \rightarrow[\phi, \psi]$ defines $E$ as a GLA. We define a homogeneous linear map $D: E \rightarrow E$ of degree 1 as follows: if $\phi \in E^{m}$, then

$$
\begin{aligned}
(D \phi)\left(x_{1}, \cdots,\right. & \left.x_{m+1}\right) \\
& =\sum_{i<j}(-1)^{i+j} \phi\left(\left[x_{i}, x_{j}\right], x_{1}, \cdots, x_{i}, \cdots, \hat{x}_{j}, \cdots, x_{m+1}\right) .
\end{aligned}
$$

\footnotetext{
1 Research supported by NSR Grants GP 1905 and GP 4503 at the University of Pennsylvania.

2 Research supported by an ONR contract at the University of Washington.
} 
Then $D$ is a derivation and $D \circ D=0$, since, for $\phi=\omega \otimes u$, we have $D=d \omega \otimes u$, where $d$ denotes the Chevalley-Eilenberg coboundary operator for cochains with values in the (trivial) $\mathfrak{g}$-module $R$ [2].

An element $\phi \in E^{1}$ is a linear map of $g$ into $\mathfrak{h}$. A simple direct computation shows that $\phi$ is a homomorphism of Lie algebras if and only if $\phi$ satisfies the "deformation equation" (see [9]) $D \phi+\frac{1}{2}[\phi, \phi]=0$. Let $R$ be the set of all homomorphisms of $\mathfrak{g}$ in to $\mathfrak{h} ; R$ is a real algebraic set in $E^{1}$. If $\rho \in R$, we define a homogeneous derivation $D_{\rho}$ of degree 1 of $E$ by $D_{\rho} \alpha=D \alpha+[\rho, \alpha]$. Then $D_{\rho} \circ D_{\rho}=0$ and hence $\left(E, D_{\rho}\right)$ is a cochain complex. A computation shows that the cohomology modules $H^{n}\left(E, D_{\rho}\right)$ are identical with the Lie algebra cohomology modules $H^{n}(\mathfrak{g}, \mathfrak{h})$ (see [2] for definitions). Here $\mathfrak{h}$ is a $\mathfrak{g}$-module via the representation $\operatorname{ad}_{\mathfrak{h}} \circ \rho$.

Let $H$ be a connected Lie group with Lie algebra $\mathfrak{h}$. (Our results are independent of the choice of $H$.) We define an $H$-module structure on $E$ as follows: if $\phi \in E^{n}$ and $h \in H$, then $h \cdot \phi=\left(\operatorname{Ad}_{\mathfrak{b}} h\right) \circ \phi$, where $\operatorname{Ad}_{\mathfrak{b}}$ denotes the adjoint representation of $H$. Then $H$ acts on the GLA $E$ by automorphisms. Thus $E$ is an analytic GLA and $D$ is an admissible derivation of $E$ in the sense of [9]; the deformation theorems stated there apply.

The set $R \subset E^{1}$ is stable under the action of $H$. Two LA homomorphisms of $\mathfrak{g}$ into $\mathfrak{h}$ are equivalent if they lie on the same orbit under $H$. A homomorphism $\rho \in R$ is rigid if the orbit $H(\rho)$ is an open subset of $R$. In this case, $H(\rho)$ is one component of a Zariski-open subset of $R$ $[9$, p. 21]. Consequently, there are only a finite number of equivalence classes of rigid homomorphisms of $\mathfrak{g}$ into $\mathfrak{h}$. The following result is a special case of [9, Theorem 18.1]:

THEOREM A. Let $\rho: \mathfrak{g} \rightarrow \mathfrak{h}$ be an LA homomorphism. If $H^{1}(\mathfrak{g}, \mathfrak{h})=0$, then $\rho$ is rigid.

A connected subset $\pi$ of $R$ containing $\rho$ is a locally complete family of deformations of $\rho$ if the orbit $H(\Re)$ is a neighborhood of $\rho$ in $R$. Theorem 20.3 of [9] on the existence of "Kuranishi families" of deformations is now applicable. Briefly, it says that there exists a locally complete family $\mathcal{K}$ of deformations of $\rho$ which can be parametrized by the set of zeros of an analytic map defined on a neighborhood of 0 in $H^{1}(\mathfrak{g}, \mathfrak{h})$ with values in $H^{2}(\mathfrak{g}, \mathfrak{h})$. If, in particular, $H^{2}(\mathfrak{g}, \mathfrak{h})$ $=0$, then $\rho$ is a simple point of $R$ (i.e., $R$ is a manifold near $\rho$ ).

We remark that all of the above results are valid with only minor modifications, if $\mathfrak{g}$ and $\mathfrak{h}$ are Lie algebras over an algebraically closed field (of arbitrary characteristic) provided that $\mathfrak{h}$ is the Lie algebra of an algebraic group $H$. 
The following theorem, which is of interest only in the case of characteristic $p>0$, is obtained by considering deformations of the identity homomorphism of a Lie algebra $\mathfrak{a}$.

TheOREM B. Let Aut(a) be the linear algebraic group of all automorphisms of a finite-dimensional Lie algebra $\mathfrak{a}$ over an infinite perfect field $k$. If $H^{2}(\mathfrak{a}, \mathfrak{a})=0$, then the Lie algebra of $\operatorname{Aut}(\mathfrak{a})$ is the Lie algebra $\operatorname{Der}(\mathfrak{a})$ of all derivations of $\mathfrak{a}$.

Here linear algebraic groups and their Lie algebras are as in Chevalley [1]. If $k$ is of characteristic 0 , the conclusion of Theorem B holds without any hypothesis on $H^{2}(\mathfrak{a}, \mathfrak{a})$; this is not the case if $k$ is of positive characteristic. A related theorem was proved for associative algebras by Gerstenhaber [5].

Exactly analogous results hold for deformations of homomorphisms of associative algebras. If $A$ and $B$ are associative algebras, then $E^{n}$ is defined to be the vector space of all $n$-linear maps of $A$ into $B$. If $\phi \in E^{m}$ and $\psi \in E^{n}$, then $[\phi, \psi] \in E^{m+n}$ is defined by

$$
\begin{aligned}
{[\phi, \psi]\left(x_{1}, \cdots, x_{m+n}\right)=} & \phi\left(x_{1}, \cdots, x_{m}\right) \psi\left(x_{m+1}, \cdots, x_{m+n}\right) \\
& -(-1)^{m n} \psi\left(x_{1}, \cdots, x_{n}\right) \phi\left(x_{n+1}, \cdots, x_{m+n}\right),
\end{aligned}
$$

which is the commutator of the "cup" product in Gerstenhaber [4]. The derivation $D$ is defined by

$$
D \phi\left(x_{1}, \cdots, x_{m+1}\right)=\sum_{i=1}^{i=m}(-1)^{i+1} \phi\left(x_{1}, \cdots, x_{i} x_{i+1}, \cdots, x_{m+1}\right) .
$$

With these modifications, all of the above results carry over for homomorphisms of $A$ into $B$; Lie algebra cohomology is replaced by the cohomology of associative algebras.

2. Relative deformations. In order to extend our results to deformations of Lie groups, we shall need a relative version of the results of $\S 1$. Let $\rho: \mathfrak{g} \rightarrow \mathfrak{h}$ be an LA homomorphism, let $\mathfrak{l}$ be a subalgebra of $\mathfrak{g}$, and consider $\mathfrak{h}$ as a $\mathfrak{g}$-module as above. Let $C(\mathfrak{g}, \mathfrak{f}, \mathfrak{h})$ be the complex used to compute the relative Lie algebra cohomology modules $H^{n}(\mathfrak{g}, \mathfrak{l}, \mathfrak{h})$ (see $[7$, p. 594] for definitions). Then $C(\mathfrak{g}, \mathfrak{l}, \mathfrak{h})$ is a graded subspace of $E$; in fact a computation shows that $C(\mathfrak{g}, \mathfrak{l}, \mathfrak{h})$ is a graded subalgebra of the GLA $E$. Moreover, $C(\mathfrak{g}, \mathfrak{h}, \mathfrak{h})$ is stable under $D_{\rho}$ and $D_{\rho}$ agrees with the standard coboundary operator on $C(\mathfrak{g}, \mathfrak{l}, \mathfrak{h})$, except for an irrelevant sign. Thus the cohomology modules of the cochain complex $\left(C(\mathfrak{g}, \mathfrak{h}, \mathfrak{h}), D_{\rho}\right)$ are just the relative cohomology modules $H^{n}(\mathfrak{g}, \mathfrak{l}, \mathfrak{h})$. 
Let $\phi \in C^{1}(\mathfrak{g}, \mathfrak{f}, \mathfrak{h})$. Then $\rho+\phi$ is a linear map of $\mathfrak{g}$ into $\mathfrak{h}$ whose restriction to $f$ agrees with $\rho$. It is trivial to see that $\rho+\phi$ is an LA homomorphism if and only if $\phi$ satisfies the deformation equation

$$
D_{\rho} \phi+\frac{1}{2}[\phi, \phi]=0 \text {. }
$$

Thus the solutions of the deformation equation correspond to the LA homomorphisms whose restriction to $l$ agrees with $\rho$. All of the results of $\$ 1$ can be carried over to such homomorphisms. We leave their detailed formulation to the reader. The group $H$ is replaced by the identity component of the subgroup $H^{\prime}$ of $H$ defined as follows: $h \in H^{\prime}$ if and only if $\left(\operatorname{Ad}_{\mathfrak{h}} h\right)(\rho(x))=\rho(x)$ for every $x \in \mathfrak{l}$.

3. Deformations of homomorphisms of Lie groups. Let $G$ and $H$ be connected Lie groups with Lie algebras $\mathfrak{g}$ and $\mathfrak{h}$. We denote by $\mathbb{R}^{\prime}$ the set of all Lie group homomorphisms of $G$ in to $H$, topologized by the compact-open topology, $R$ is as in $\$ 1$. Let $\theta: \mathcal{R}^{\prime} \rightarrow \operatorname{Hom}_{R}(\mathfrak{g}, \mathfrak{h})$ be the map which assigns to each homomorphism $r: G \rightarrow H$ the corresponding LA homomorphism $d r: \mathfrak{g} \rightarrow \mathfrak{h}$. Set $\mathcal{R}^{\prime \prime}=\theta\left(\mathcal{R}^{\prime}\right)$. Then $\theta$ is a homeomorphism of $R^{\prime}$ onto $R^{\prime \prime}$ and $R^{\prime \prime}$ is an analytic subset of $\operatorname{Hom}_{R}(\mathfrak{g}, \mathfrak{h})$. This allows us to use differential-geometric reasoning in studying $\mathbb{R}^{\prime}$.

If $h \in H$, let $I_{h}$ denote the corresponding inner automorphism of $H$. We define $R^{\prime}$ as a topological transformation space for $H$ by setting $h \cdot r=I_{h} \circ r$ for $h \in H$ and $r \in \Omega^{\prime}$. Then $\theta$ is equivariant with respect to the actions of $H$ on $\mathcal{R}^{\prime}$ and $\operatorname{Hom}_{\boldsymbol{R}}(\mathfrak{g}, \mathfrak{h})$. Two homomorphisms of $G$ into $H$ are equivalent if they lie on the same orbit under the action of $H$. A homomorphism $r$ is rigid if the orbit $H(r)$ is an open subset of $\Omega^{\prime}$.

For Lie groups, we have the following analogue of Theorem A. We refer the reader to [6] for the (differentiable or, equivalently, continuous) cohomology theory of Lie groups.

THEOREM C. Let $r: G \rightarrow H$ be a homomorphism of connected Lie groups and consider $\mathfrak{h}$ as a G-module via the representation $\mathrm{Ad}_{\mathfrak{b}} \circ \mathrm{r}$. If $H^{1}(G, \mathfrak{h})=0$, then $r$ is rigid.

The proof is similar to that given by Weil in $[10$, p. 152] for discrete $G$.

Let $C$ be a maximal compact subgroup of $G$, and let $c$ be the Lie algebra of $C$. If $V$ is a $G$-module, then it is known [6] that $H^{n}(C, V)$ $=0$ for $n>0$ and that $H^{n}(G, V)$ is canonically isomorphic to the relative LA cohomology $H^{n}(\mathfrak{g}, \mathfrak{c}, V)$.

Let $r \in \mathcal{R}^{\prime}$ and let $\rho=d r$. We consider $\mathfrak{h}$ as a $G$-module (respectively $\mathfrak{g}$-module) as above. Let

$$
R_{C}^{\prime}=\left\{s \in \mathcal{R}^{\prime} \mid s(x)=r(x) \text { for every } x \in C\right\} .
$$


We define $R_{c}$ similarly. We now apply Theorem $C$ to deformations of homomorphisms of $C$ into $H$. Since $H^{1}(C, \mathfrak{h})=0$, it follows that there exist a neighborhood $N^{\prime}$ of $r$ in $\mathbb{R}^{\prime}$ and a continuous map $\gamma: N^{\prime} \rightarrow H$ with $\gamma(r)=e$ (e denotes the identity element of $H$ ) such that $\gamma(s) \cdot s$ $\in \Omega_{C}^{\prime}$ for every $s \in N^{\prime}$. Furthermore, an argument involving the simply connected covering group of $G$ shows that there exists a neighborhood $N$ of $\rho$ on $\mathfrak{R}_{\mathfrak{c}}$ such that, if $\tau \in N$, then there exists $s \in N^{\prime}$ such that $d s=\tau$. Let $\Re$ be a (sufficiently small) Kuranishi family for $\rho$ on $\mathfrak{R}_{\mathfrak{c}}$. (KK exists by the results of $\S 2$.) Let $\mathfrak{K}^{\prime}=\theta^{-1}(\mathcal{K})$. Then it follows easily that $\varkappa^{\prime}$ is a locally complete family of deformations of $r$. Since $H^{n}(G, \mathfrak{h})=H^{n}(\mathfrak{g}, \mathfrak{c}, \mathfrak{h})$, we see that $\mathfrak{K C}^{\prime}$ can be parametrized by the set of zeros of an analytic map defined on a neighborhood of 0 in $H^{1}(G, \mathfrak{h})$ with values in $H^{2}(G, \mathfrak{h})$. Thus $\mathcal{K}^{\prime}$ is a Kuranishi family for $r$ in $\mathbb{R}^{\prime}$.

Remarks. (1) It is not difficult to extend Theorem $C$ to the case in which $G$ is a Lie group whose group of components is finitely generated and $H$ is an arbitrary Lie group. However, we have not been able to extend the results on Kuranishi families to this case.

(2) Let $G$ be a compact topological group. J. M. G. Fell has outlined to one of the authors a proof that every finite-dimensional representation of $G$ is rigid. More generally, we conjecture that Theorem C is valid if $G$ is a compactly generated locally compact topological group and $H$ is a Lie group.

\section{REFERENCES}

1. C. Chevalley, Theorie des groupes de Lie. II, Groupes algebriques, Hermann, Paris, 1951.

2. C. Chevalley and S. Eilenberg, Cohomology theory of Lie groups and Lie algebras, Trans. Amer. Math. Soc. 63 (1948), 85-124.

3. A. Frölicher and A. Nijenhuis, $A$ theorem on stability of complex structures, Proc. Nat. Acad. Sci. U.S.A. 43 (1957), 239-241.

4. M. Gerstenhaber, The cohomology of an associative ring, Ann. of Math. 78 (1963), 267-289.

5. M. Gerstenhaber, On the deformation of rings and algebras, Ann. of Math. 79 (1964), 59-104.

6. G. Hochschild and G. D. Mostow, Cohomology of Lie groups, Illinois J. Math. 6 (1962), 36-401.

7. G. Hochschild and J. P. Serre, Cohomology of Lie algebras, Ann. of Math. 57 (1953), 591-603.

8. M. Kuranishi, On the locally complete families of complex analytic structures, Ann. of Math. 75 (1962), 536-577.

9. A. Nijenhuis and R. Richardson, Cohomology and deformations in graded Lie algebras, Bull. Amer. Math. Soc. 72 (1966), 1-29. 158.

10. A. Weil, Remarks on the cohomology of groups, Ann. of Math. 80 (1964), 149-

University of Pennstluania ANd

UNIVERSITY OF WASHINGTON 